a slight muco-purulent discharge. In other cases there was no pain in the ear and only a slight discharge, and the men, thinking it only trivial, did not complain until it became profuse and was accompanied by marked deafness.

During the last twelve months I have examined some hundreds of cases of deafness in varying degree for pension purposes, and I now, as never before, realize at what a disadvantage a man with even a partial loss of hearing is. There are many trades in which good hearing is indispensable. The miner has again and again told me he can no longer get work down the pit on account of his loss of hearing, because he may fail to hear sounds in the pit which portend disaster or death to himself or his com. rades. The same applies to many other trades, and the man has to seek other work, each succeeding class of work is more precarious for him, and he is finally driven to casual labour. The poorer classes take but casual notice of the condition of the ear, and this applies especially to suppurative otitis media. Pain is not a frequent concomitant, otherwise they would oftener seek advice. I cannot do better than quote Dr. Mygind of Copenhagen in his book on deaf-mutism. He writes: "It is to be hoped that the recognition which is by degrees, though slowly, being yielded to otology by the medical profession, will make itself felt in the prevention of deaf-mutism, by opening the eyes of the practitioner to the importance of ear diseases and their treatment, and also that the general public may be led to form other opinions upon the subject than those now prevalent."

If by propaganda the poor and so-called working classes could be taught to look upon their hearing as precious, and realize that a "running ear" may lead to the loss of that power and calls at the earliest onset for advice, the efficiency of the nation in general would be increased.

\section{STRANGULATED UMBILICAL HERNIA :}

Resection of Gangrenous Ileum at the Age of 69. BY

O. M. KENNEDY, M.B.E., F.R.C.S.ENG., LATE MAJOR R.A.M.C.(T.C.),

ASBISTANT SURGEON SOUTH DEVON AND EAST CORNWALL HOSPITAL,

Successfur resection of gangrenous gut in inguinal and femoral herniae are by no means rare, but in umbilical herniae successful resection is less common. The following case is thought to be of interest because success was attained in spite of the patient's age.

M. P., a widow aged 69, was admitted to the South Devon and East Cornwall Hospital on the afternoon of January 2lst, 1920, complaining of severe abdominal pain and vomiting.

Her history was difficult to elicit, and was probably inaccurate. So far as could be discovered she had had an umbilical hernia for many years. She stated that she had had pain in the hernia for more than a week, associated with vomiting. During this time the bowels had been very slightly opened after aperients, and for three or four days had not moved at all. She did not call a doctor in until the day of moved at all. She did not call a doctor in

On admission she looked ill, and senile beyond her years. The pulse was 94 but weak, the temperature $98^{\circ}$. At the umbilicus was a tense tender red-almost purple-swelling about the size of a cricket ball. There was no impulse on coughing. The rest of the abdomen was somewhat distended, but not tender. The vomit was bile-stained and foul, but hardly "faecal."

Under a chloroform and ether mixture the hernia was surrounded by a transverse elliptical incision, which was deepened to the rectus sheath. The hernis was then lifted off the rectus sheath until the neck was exposed all round. The abdomen was then opened just clear of the neck of the hernia. The hernia was then isolated by extending this incision completely round, but just clear of, the neck. The abdomen was then packed off, and the sac opened by splitting up the neck from what had been its peritoneal aspect. The sac contained a quantity of very fouf, almost black, purulent fluid, a mass of adherent and gangrenous omentum, which was ligatured of and removed with the sac, and about four inches of gangrenous lleum. The centre of this length of small gut was of very doubtful viability, but at either end, where it had been nipped, it was reduced to little more than a slough. In order to leave a good margin on either side of the damaged gut about eight or nine inches of gut was resected. Continuity was restored by end to end anastomosis, the collapsed distal portion of the gut being split along its anti-mesenterio border so as to give a sufficient lumen for an anastomosis to the proximal distended gut.
The abdomen was closed in layers by mattress sutures placed transversely (modifled Mayo's method). A rubber drain was placed at either angle of the wound, as the subcutaneous fat was considered likely to be infected.

On the day following operation the temperature was $97.4^{\circ}$, the pulse 80 , and respirations 22 . There was considerable flatulence. The bowels were opened by a turpentine enema on the following day. Thereafter convalescence was smooth but for trifling superficial sepsis necessitating removal of a couple of skin sutures.

The patient left hospital for a convalescent home on February $24 \mathrm{th}, 1920$. She was then in her normal state of health.

\section{fftemorattò:}

\section{MEDICAL, SURGICAL, OBS'TE'TRICAL.}

\section{SHINGLES AND CHICKEN-POX.}

Acmost immediately after reading Dr. Taylor's neurological jottings in your issue of February 28th, a girl of 16 came to my surgery for some medicine for her father, who had developed shingles about a fortnight before. She asked me about a " rash" which had appeared on her body on the previous day. I found that she was in an early stage of chicken-pox, which subsequently developed, with a temperature of $102^{\circ}$, into a good crop of vesicles. There is no other case of chicken-pox in the neighbourhood. Her father is an instructor in gardening at a boys' home, but there have been no cases amongst the boys, probably because of the work being entirely in the open air.

Chobham. Surrey. A. Ingram Cooke, M.D.

\section{ACUTE OEDEMA OF THE LUNGS.}

I HAVE been much interested in the correspondence on this subject, as, with Dr. Stewart McNaughton (February 28th, p. 293) I believe that the disease is more common than is usually thought. In the autumn of $1897 \mathrm{I}$ reported \& case of dramatic severity in the Lancet.

The victim was a man aged about 50 , who was seized with respiratory distress a fter a bicycle ride against a cold head wind. He died within an hour of his first symptoms, and for the last few minutes of his life poured out pints of 'bright' pink foam. Auscultation revealed the presence of fine orepitant râles, com mencing at the bases and very rapidly spreading upwards. There was a history of some previous renal mischief.

Within a few months I saw a woman of much the same age who fell ill suddenly after cleaning out a flooded basement. Her condition was less acute. The expectoration was aërated, bright pink, but not so great in quantity, nor so frothy. She lived o few hours

Aguin, a man of 60 was placed under my care with a story of recurring suffocative attacks at night. He had aortic regurgita tion. The urine was of low gravity, and contained a trace of albumin. During a period of perhaps two months he called me some half-dozen times; always a little before midnight. He was invariably in great distress, standing with both hands grasping the bed rail. The sputum was more or less frothy and coloured red. A hypodermic injection of atropine would relieve him at once, allow him to fo back to bed, and in the morning he would be quite recovered. The fine relle was very morning he would be quite recovered. The fine rale was very evident in this case, and the rapidity of the upward extensian
remarkable. His last attack was more severe and I was longer in reaching him. This time atropine failed to check the secretion, and he died expelling much frothy sputum.

Two other cases of recurrent oedema occur to me. One in a woman of over 70 , suffering from mitral regurgitation; the other in a woman of 60 , suffering from myocardial degeneration. In each instance the crises were spread over a period of two or three years at varying intervals of months. In each there was the great distress, the rapidly ascending râle, and the tinged aërated sputum. Atropine acted well in both and promptly relieved the condition.

The older patient eventually, after her cardiac condition had badly deteriorated, died in an attack, in spite of the usual dose. The other died of gradual heart failure and generalized oedema. The last time $I$ saw the condition was in the following case:

A man of 46. He suffered from extreme arterial high tension, and gave a history of a former but milder bout.

I was called in the night to find him intensely dyspnoeic unable to move or lie down. - There was the usual type of sputum; but for the first time in my experience the riles were the most abundant at the upper part of the lungs. A dose of atropine relieved at once and allowed him to return to bed. In the morning he was quite well.

There are differences in all these cases, but they chearly belong to one group. It is noticeable that where history 